\title{
Lattice Boltzmann Phase Field Simulations of Droplet Slicing
}

\begin{tabular}{|c|c|}
\hline Journal: & The Canadian Journal of Chemical Engineering \\
\hline Manuscript ID & CJCE-20-0797.R1 \\
\hline Wiley - Manuscript type: & Article \\
\hline $\begin{array}{r}\text { Date Submitted by the } \\
\text { Author: }\end{array}$ & $11-J a n-2021$ \\
\hline Complete List of Authors: & $\begin{array}{l}\text { Lu, Zeying; Shanghai University of Engineering Science, School of } \\
\text { Chemistry and Chemical Engineering } \\
\text { Liu, Fengjiao; Shanghai University of Engineering Science, School of } \\
\text { Chemistry and Chemical Engineering } \\
\text { Liu, Xijian; Shanghai University of Engineering Science, School of } \\
\text { Chemistry and Chemical Engineering } \\
\text { Zhang, Lijuan; Shanghai University of Engineering Science, School of } \\
\text { Chemistry and Chemical Engineering } \\
\text { Yuan, Haikuan; Shanghai University of Engineering Science, School of } \\
\text { Chemistry and Chemical Engineering } \\
\text { Lu, Jie; Shanghai University of Engineering Science, School of Chemistry } \\
\text { and Chemical Engineering } \\
\text { Derksen, Jacobus; University of Aberdeen, School of Engineering }\end{array}$ \\
\hline Keywords: & $\begin{array}{l}\text { droplet slicing, free energy model, lattice-Boltzmann method, rising } \\
\text { droplet }\end{array}$ \\
\hline
\end{tabular}




\section{Highlights:}

1. We performed 2D and 3D lattice Boltzmann phase field simulations of rising drops

2. The rising drops are sliced by a knife, we study this process and the fate of the daughter drops

3. We identify a critical Eotvos number that needs to be exceeded for proper slicing

4. We correlate volume ratios of asymmetrically sliced drops with off-center distances of the knife 


\title{
Lattice Boltzmann Phase Field Simulations of Droplet Slicing
}

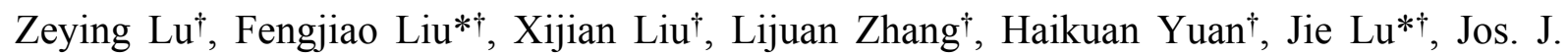
Derksen

†School of Chemistry and Chemical Engineering, Shanghai University of Engineering Science, Shanghai, China

SSchool of Engineering, University of Aberdeen, Aberdeen, UK

Correspondence

Fengjiao Liu, School of Chemistry and Chemical Engineering, Shanghai University of Engineering Science, Shanghai, China.Email: fjliu@sues.edu.cn

Jie Lu, School of Chemistry and Chemical Engineering, Shanghai University of Engineering Science, Shanghai, China. Email: lujie@sues.edu.cn

\begin{abstract}
We have performed two and three-dimensional phase field simulations using the lattice-Boltzmann method of liquid drops rising through a continuous phase liquid under the influence of buoyancy. In their upward motion the drops encounter a knife that is placed with the purpose of slicing the drops in two. A range of scenarios has been observed when the drop hits the knife and it has been investigated how the type of scenario depends on the dimensionless parameters governing the motion and slicing of the drop: the Eötvös number, the Morton number and the ratio of the droplet size and the width of the knife. We studied symmetric and asymmetric encounters between drop and knife and kept track of the size distribution of the resulting fragments.
\end{abstract}

Keywords

droplet slicing, free energy model, lattice-Boltzmann method, rising droplet 


\section{INTRODUCTION}

Emulsions - systems of two or more immiscible liquids in the form of droplets dispersed in a continuous phase - are an essential part of many engineering processes and products. Controlling and influencing the drop sizes and drop size distributions often is essential for product quality and process efficiency. The desired direction of change of droplet size depends on our objectives. Increasing droplet size through coalescence could be helpful for gravity driven phase separation as in oil-water separation devices. ${ }^{[1-3]}$ Reducing the droplet size increases interfacial area which would benefit mass transfer between phases in, for instance, extraction operations. ${ }^{[4-5]}$ Another example is herbicide toxicity which will increase when its droplets get smaller with smaller droplets creating a larger number of contact points on the plant compared to larger droplets. ${ }^{[6]}$

In this paper we will be exploring size reduction of droplets by slicing them. Applications of slicing (or cutting) droplets are mainly found in microfluidics ${ }^{[7]}$ and also - on a larger scale - in static mixers. ${ }^{[8]}$ Forte et al ${ }^{[9]}$ investigated the fundamental mechanisms of oil droplet breakup in static mixers. They found that the drop size decreased to a critical point, when the continuous phase (water) flow rate was increasing. It became difficult to reduce the oil drop size beyond the critical value. Additionally, Jaworski et al ${ }^{[10]}$ modelled the process of droplet breakup in a static mixer and predicted the drop size evolution as a result of breakup. They found that intensive drop breakage occurred inside the inserts resulting in a decrease of the average drop size. Ren et al ${ }^{[11]}$ investigated the droplet breakup dynamics through a bi-layer bifurcating microchannel by numerical and experimental method. In the works cited above the drop motion is the result of a pressure driven flow, bounded by walls. In our paper we study slicing of a freely rising drop in a periodic domain without bounding walls. This is a relatively simple system that, however, allows us to relate the starting point of our simulations (a rising drop) to extensively available on drop rise velocities and drop shapes. ${ }^{[12]}$

Since the 1970s, a computational fluid dynamics (CFD) method based on solving the Reynolds-averaged Navier-Stokes (RANS) equation has been widely used for computing fluid flow and heat transfer. ${ }^{[13]}$ In the last 25 years, the lattice Boltzmann method (LBM) has been an efficient and powerful simulation method for various of transport phenomena and processes, ${ }^{[13-17]}$ such as single-phase flow, multi-phase flows, turbulence, heat transfer, and 
phase change, also become a numerical tool to solve nonlinear partial differential equations. ${ }^{[18-21]}$ It has many unique benefits compared with other numerical methods.[22] Unlike traditional methods, LBM is based on the perspective of micro-dynamics, taking macro physical quantities as the result of statistical averages of micro quantities. LBM has both microscopic and mesoscopic characteristics. It makes the interaction between particles easy to resolve, and enables complex macroscopic phenomena of multiphase flow to appear naturally, thus providing an effective way to study the mechanism of multiphase flow under complex conditions.

There have been many studies using LBM to simulate droplet behaviour, including the droplet formation in micro-channels, ${ }^{[16]}$ deformation and breakup of a droplet exposed to a gas stream, ${ }^{[23]}$ three-dimensional binary droplet collisions ${ }^{[24]}$ etc. In addition to these, $\mathrm{Yu}$ et $\mathrm{al}^{[25]}$ firstly addressed the dynamics of droplets in a static mixer using obstacles and porous media by means of a modified LBM. They found that there is a critical obstacle size, beyond which an obstacle is unfavorable for generating small droplets.

The behaviour of droplet rising through liquid driven by buoyancy has been observed by experiment and numerical simulations. Bertakis et al ${ }^{[26]}$ studied the binary liquid system (n-butanol/water) by experiment and numerical simulations. Watanabe and Ebihara ${ }^{[27]}$ used two-component two-phase LBM to simulate the process of drop rising. Komrakova et al[28] performed numerical simulation of n-butanol drop rising in water using the multiphase LBM, the simulation results they obtained agree well with the experimental data.

The focus of this study is the slicing of a rising droplet by a thin, flat plate - a knife. It is the aim of this study to investigate the feasibility of a knife as an efficient and controllable way of reducing the droplet size and to assist the design of process equipment for this purpose. We expect that the quality of the slicing process depends on the forces involved - buoyancy, surface and viscous forces - as well as the geometrical details such as the sharpness and length of the knife. The diffuse interface free energy LBM model will be used. ${ }^{[29]}$

\section{FLOW CONFIGURATION \& FLOW CONDITIONS}

Numerical simulation of slicing a rising droplet is the focus of the present study. A liquid drop of density $\rho_{d}$ and dynamic viscosity $\mu_{d}$ with equivalent diameter $d$ is suspended in 
another liquid of density $\rho_{c}$ and dynamic viscosity $\mu_{c}$ (subscripts $d$ and $c$ represent dispersed and continuous phase). The density difference between the two liquids is $\Delta \rho=\rho_{c}$ $-\rho_{d}>0$. The interfacial tension between the liquids is $\sigma$. At time $t=0$, the drop is motionless. The droplet begins to rise driven by gravity and buoyancy and eventually attains a terminal rise velocity $u$. Table 1 gives the physical parameters for binary benchmark system.

Table 1. Physical parameters for the simulated system

\begin{tabular}{cc}
\hline density & $\rho_{c}=1 \times 10^{3} \mathrm{~kg} / \mathrm{m}^{3}$ \\
\hline density difference & $\Delta \rho=1 \times 10^{2} \mathrm{~kg} / \mathrm{m}^{3}$ \\
dynamic viscosity & $\mu_{c}=3.34 \times 10^{-3} \mathrm{Pas}$ \\
dynamic viscosity & $\mu_{d}=3.34 \times 10^{-3} \mathrm{Pas}$ \\
surface tension & $\sigma=1 \times 10^{-3} \mathrm{~N} / \mathrm{m}$ \\
\hline
\end{tabular}

Along with gravitation acceleration $g$ the input parameters translate in four dimensionless groups that fully determine the problem. In this paper we take for these dimensionless numbers the Eötvös number $E o=\frac{\mathrm{g} \Delta \rho d^{2}}{\sigma}$, the Morton number $M o=\frac{\mathrm{g} \Delta \rho v_{c}^{4} \rho_{c}^{2}}{\sigma^{3}}=\frac{\mathrm{g} \Delta \rho \mu_{c}^{4}}{\rho_{c}^{2} \sigma^{3}}$, the density ratio $\lambda=\frac{\rho_{d}}{\rho_{c}}$, and the dynamic viscosity ratio $\eta=\frac{\mu_{d}}{\mu_{c}}$.

Dimensionless numbers that involve the rise velocity are output parameters: the Reynolds number $R e=\frac{u d \rho_{c}}{\mu_{c}}$, the Weber number $W e=\frac{\rho_{c} u^{2} d}{\sigma}$, and the Froude number $F r=\frac{u^{2}}{\mathrm{~g} d}$. They characterize the relative importance of inertia over viscous effects $(R e)$, inertia and surface forces $(W e)$ and inertia and gravity / buoyancy forces $(F r)$. Eo quantifies the ratio of gravity / buoyancy and surface forces. Mo is a constant for a given system, it does not contain the drop size.

An important reference point in our work is the diagram by Clift et al[12] that allows one to determine the Reynolds number (output parameter) as well as the drop shape and its rising regime (steady, wobbling...) from $E o$ and $M o$ (input parameters).

We emphasize the importance of dimensionless numbers given that we use the LBM that is operated with its own "lattice units" with length and time units the lattice spacing and time 
step respectively. Translating between physical (SI) units and lattice units makes use of the dimensionless numbers: if a physical system and a numerical (LBM) system have the same input dimensionless numbers, they represent the same system. In this paper, parameters in lattice units will be given a $\sim$. For example, the drop diameter in lattice units is denoted as $\tilde{d}$ which is the number of lattice spacings covered by the drop diameter. Unit conversion / scaling has been discussed in detail in Section 3.3.

The base-case simulation domain was a cuboid of $20 \tilde{d} \times 3 \tilde{d} \times 1$ (for two-dimensional simulation) and $20 \tilde{d} \times 3 \tilde{d} \times 3 \tilde{d}$ (for three-dimensional simulation). As shown in Figure 1, the "knife" was placed at the top of the simulation domain. Initially it was located in the top-centre with the drop released on the centre line so that it was cut through the middle. Then the knife was moved laterally to study what happens if the drop hits the knife off-centre (see the details given in Section 5.2). Figure 1 also defines a Cartesian coordinate system as used throughout this paper.

To complete the definition of the flow system, boundary conditions have to be specified. Periodic conditions applied on all outer faces. No-slip conditions were imposed on the knife, and we assume a $90^{\circ}$ contact angle on the surface of the knife.

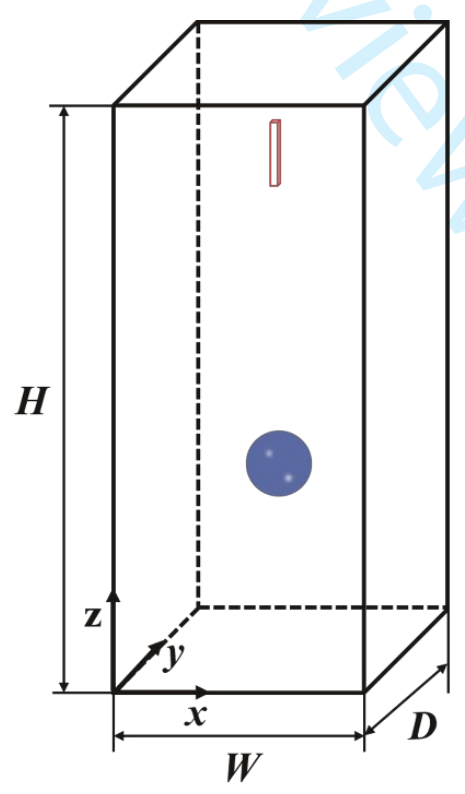

Figure 1. Simulation domain with a "knife" at the top and the droplet released on the centre line 


\section{SIMULATION METHOD}

\subsection{Governing equations}

In this study, we use the diffuse interface model for simulation of flow and interface dynamics. In diffuse interface (which are also called phase field) methods, ${ }^{[30-32]}$ we use a thin transition layer to represent the phase interface. An order parameter $\phi$ is used to describe the composition of system. It is a function of position vector $\boldsymbol{r}$ and time $t$. The value of $\phi$ is constant in the bulk phase of each fluid and varies continuously in the range $[-1,1]$ over the thin interface.

Order parameter $\phi$ represents the relative concentration of the two components for the system when describing behaviour of binary system by phase field. ${ }^{[33-34]}$ Cahn and Hilliard ${ }^{[34-35]}$ proposed that continuity, momentum and convection-diffusion equations can be used in combination for the simulation of fluid dynamics:

$$
\begin{aligned}
& \partial_{\mathrm{t}} \rho+\partial_{\alpha}\left(\rho \mathrm{u}_{\alpha}\right)=0 \\
& \quad \partial_{\mathrm{t}}\left(\rho \mathrm{u}_{\alpha}\right)+\partial_{\beta}\left(\rho \mathrm{u}_{\alpha} \mathrm{u}_{\beta}\right)=-\partial_{\beta} \mathrm{P}_{\alpha \beta}^{\mathrm{th}}+\partial_{\beta} v\left(\rho \partial_{\alpha} \mathrm{u}_{\beta}+\rho \partial_{\beta} \mathrm{u}_{\alpha}\right)+\mathrm{g}_{\alpha} \Delta \rho \\
& \partial_{\mathrm{t}} \phi+\partial_{\alpha}\left(\phi \mathrm{u}_{\alpha}\right)=\mathrm{M} \partial_{\beta \beta}^{2} \mu
\end{aligned}
$$

where $\rho$ is density, and $v$ is kinematic viscosity of the mixture, the index $\alpha$ stands for the Cartesian direction $x, y$ or $z$ so that $\mathrm{u}_{\alpha}$ is the velocity component in $\alpha$ direction and $\mathrm{g}_{\alpha}$ is the component of gravitational acceleration. The parameter $M$ is the mobility parameter. Here $\mathrm{P}_{\alpha \beta}^{\mathrm{th}}$ is the "thermodynamic" pressure tensor, which contains two parts ${ }^{[36]}$ : an isotropic contribution $P \delta_{\alpha \beta}$ that describes the ideal gas pressure and the "chemical" pressure tensor $P_{\alpha \beta}^{\text {chem }}$ defined by the following:

$$
P_{\alpha \beta}^{\text {chem }}=\delta_{\alpha \beta}\left(\phi \frac{\delta V}{\delta \phi}-V-\kappa\left(\phi \partial_{y y}^{2} \phi+\frac{1}{2}\left|\partial_{\alpha} \phi\right|^{2}\right)\right)+\kappa\left(\partial_{\alpha} \phi\right)\left(\partial_{\beta} \phi\right)
$$

Phase separation is described by symmetrical double well potential, we can find this by the following:

$$
V=\frac{A}{2} \phi^{2}-\frac{A}{4} \phi^{4}
$$

The chemical potential $\mu(\phi)$ in Equation (1c) can be seen as follows ${ }^{[37]}$ :

$$
\mu(\phi)=\mathrm{A} \phi-\mathrm{A} \phi^{3}-\kappa \partial_{\alpha \alpha}^{2} \phi
$$


where, $A<0$, both $A$ and $\kappa$ are parameters connected with surface tension and interface thickness as explained in Section 3.2.

Given the relatively minor density difference between continuous phase and disperse phase liquid (10\%, see Table 1) we apply the Boussinesq approximation ${ }^{[38]}$ which can be applied for flows with relatively small density differences. This means that the density in Equation (1a) and (1b) is uniform $\left(\rho=\rho_{c}\right)$ and the density difference between drop and surroundings only has an effect in the forcing term $\mathrm{g}_{\alpha} \Delta \rho$ in momentum Equation (1b).

\subsection{LBM implementation}

The free energy model was proposed by Swift et al ${ }^{[29]}$ to solve the system of Equations (1). This model is adopted in the present study. Two particle distribution functions are utilized: one function $f(\boldsymbol{r}, t)$ is used to solve the continuity (1a) and the Navier-Stokes $(1 \mathrm{~b})$ Equations and the second one $g(\boldsymbol{r}, t)$ is used for the convection-diffusion Equation (1c). The distribution functions evolve with discrete time steps $\Delta t$. All simulations were performed using a single relaxation time collision operator (known as Bhatnagar-Gross-Krook (BGK) model). ${ }^{[39]}$ The discretized LBEs are given as following:

$$
\begin{aligned}
& f_{q}\left(r_{\alpha}+c_{\alpha q} \Delta t, t+\Delta t\right)=f_{q}\left(r_{\alpha}, t\right)-\frac{f_{q}(r, t)-f_{q}^{e q}(r, t)}{\tau_{f}}+F_{q} \\
& g_{\mathrm{q}}\left(r_{\alpha}+c_{\alpha q} \Delta t, t+\Delta t\right)=g_{q}\left(r_{\alpha}, t\right)-\frac{g_{q}(r, t)-g_{q}^{e q}(r, t)}{\tau_{g}}
\end{aligned}
$$

where index $q$ is discretized velocity direction; $f_{q}\left(r_{\alpha}+c_{\alpha q} \Delta t, t+\Delta t\right)$ and $g_{q}$ $\left(r_{\alpha}+c_{\alpha q} \Delta t, t+\Delta t\right)$ are post-collision particle distribution functions streamed from $\left(r_{\alpha}, t\right)$ to $\left(r_{\alpha}+c_{\alpha q} \Delta t, t+\Delta t\right)$ along direction $q ; f_{q}\left(r_{\alpha}, t\right)$ and $g_{\mathrm{q}}\left(r_{\alpha}, t\right)$ represent pre-collision particle distribution functions; $f_{q}^{e q}, g_{\mathrm{q}}^{\mathrm{eq}}$ are the discrete Maxwell-Boltzmann distributions (or equilibrium distributions); $c_{\alpha q}$ denotes the discrete velocity set and $c=\Delta x / \Delta t$ is the lattice speed; $\tau_{f}$ and $\tau_{g}$ are dimensionless relaxation parameters, $\tau_{f}$ is determined by the kinematic viscosity, as follows:

$$
\tau_{f}(\phi)=\frac{v(\phi)}{c_{s}^{2} \Delta t}+\frac{1}{2}
$$

where $c_{s}^{2}=\frac{1}{3}$ represents the square of sound speed in lattice units. 
The relationship between mobility $M$, coefficient of mobility $\Gamma$, and relaxation parameter $\tau_{g}$ is given by ${ }^{[37]}$ :

$$
M=\Delta t \Gamma\left(\tau_{g}-\frac{1}{2}\right)
$$

where the order parameter mobility $M$ is determined by the parameter $\Gamma$ as well as relaxation parameter $\tau_{g} . F_{q}$ is the forcing term.

The D3Q19 lattice is adopted here to discretize the velocity space. ${ }^{[40]}$ Each site has 19 velocity directions.

As noted above, the LBM works in dimensionless lattice units. We utilized uniform cubic lattices for the method described here. The mesh step $\Delta x$ is unit of length, and the time step $\Delta t$ is the unit of time. Here, we give the discrete velocity set:

$$
\left(\begin{array}{c}
c_{x} \\
c_{y} \\
c_{z}
\end{array}\right)=\left[\begin{array}{ccccccccccccccccccc}
0 & c & -c & 0 & 0 & 0 & 0 & c & -c & c & -c & 0 & 0 & 0 & 0 & c & -c & c & -c \\
0 & 0 & 0 & c & -c & 0 & 0 & c & c & -c & -c & c & -c & c & -c & 0 & 0 & 0 & 0 \\
0 & 0 & 0 & 0 & 0 & c & -c & 0 & 0 & 0 & 0 & c & c & -c & -c & c & c & -c & -c
\end{array}\right]
$$

The local density, local fluid momentum, and local order parameter of the fluid at lattice point are the results of summations over all directions $q$ at that location, these can be seen as follows:

$$
\begin{aligned}
& \sum_{q} f_{q}=\rho \\
& \sum_{q} \boldsymbol{c}_{q} f_{q}=\rho \boldsymbol{u}+\frac{\Delta t}{2} \boldsymbol{F} \\
& \sum_{q} g_{q}=\phi
\end{aligned}
$$

The forcing term is incorporated as follows:

$$
F_{q}=\omega_{q}\left(\boldsymbol{c}_{q} \cdot \boldsymbol{F}\right)
$$

here $\boldsymbol{F}$ represents body force $\mathbf{g} \Delta \rho, \omega_{q}$ are weight coefficients. ${ }^{[41]}$ Additional weighing coefficients $\omega_{q}^{\alpha \beta}$ are used for the equilibrium distributions, we can determine them by the following (see Equation (14)):

$$
\begin{aligned}
& \omega_{0}=1, \omega_{1-6}=\frac{1}{6}, \omega_{7-18}=\frac{1}{12} \\
& \omega_{1-2}^{x x}=\omega_{3-4}^{y y}=\omega_{5-6}^{z z}=\frac{5}{12}, \omega_{3-6}^{x x}=\omega_{1-2,5-6}^{y y}=\omega_{1-4}^{z z}=-\frac{1}{3} \\
& \omega_{7-10}^{x x}=\omega_{15-18}^{x x}=\omega_{7}^{y y}{ }_{-14}=\omega_{11-18}^{z z}=-\frac{1}{24}
\end{aligned}
$$


$\omega_{11-14}^{x x}=\omega_{15-18}^{y y}=\omega_{7-10}^{z z}=\frac{1}{12}$

(10)

$$
\begin{aligned}
& \omega_{1-6}^{x y}=\omega_{1-6}^{y z}=\omega_{1-6}^{z x}=0, \omega_{7,10}^{x y}=\omega_{11,14}^{y z}=\omega_{15,18}^{z x}=\frac{1}{4} \\
& \omega_{8-9}^{x y}=\omega_{12-13}^{y z}=\omega_{16-17}^{z x}=-\frac{1}{4}, \omega_{11-18}^{x y}=\omega_{7-10}^{y z}=\omega_{7-14}^{z x}=0
\end{aligned}
$$

The full pressure tensor $P_{\alpha \beta}$ is defined by the following:

$$
P_{\alpha \beta}=\sum_{q} f_{q} c_{q \alpha} c_{q \beta}
$$

The equilibrium distributions must satisfy conditions (8) and $\phi$ is advected by fluid, which can be given by the follow equation:

$$
\sum_{q} g_{q}^{e q} c_{q \alpha}=\phi u_{\alpha}
$$

the pressure tensor and chemical potential at equilibrium follow:

$$
\begin{aligned}
& \sum_{q} f_{q}^{e q} c_{q \alpha} c_{q \beta}=P_{\alpha \beta}^{t h}+\rho u_{\alpha} u_{\beta} \\
& \sum_{q} g_{q}^{e q} c_{q \alpha} c_{q \beta}=\Gamma \mu \delta_{\alpha \beta}+\phi u_{\alpha} u_{\beta}
\end{aligned}
$$

The equilibrium distributions ${ }^{[41]} f_{q}^{e q}, g_{\mathrm{q}}^{\mathrm{eq}}$ for populations $1 \leq q \leq 18$ are calculated using the following equations:

$$
\begin{gathered}
f_{q}^{e q}=\frac{\omega_{q}}{c^{2}}\left(p_{0}-\kappa \phi\left(\partial_{x x}^{2} \phi+\partial_{y y}^{2} \phi+\partial_{z z}^{2} \phi\right)+c_{\alpha q} \rho u_{\alpha}+\frac{3}{2 c^{2}}\left(c_{\alpha q} c_{\beta q}-\frac{c^{2}}{3} \delta_{\alpha \beta}\right) \rho u_{\alpha} u_{\beta}\right. \\
+\frac{\kappa}{c^{2}} \\
\left(\omega_{q}^{x x} \partial_{x} \phi \partial_{x} \phi+\omega_{q}^{y y} \partial_{y} \phi \partial_{y} \phi+\omega_{q}^{z z} \partial_{z} \phi \partial_{z} \phi+\omega_{q}^{x y} \partial_{x} \phi \partial_{y} \phi+\quad \omega_{q}^{x z} \partial_{x} \phi \partial_{z} \phi+\partial_{y} \phi \partial_{z} \phi\right)
\end{gathered}
$$

$$
g_{q}^{e q}=\frac{\omega_{q}}{c^{2}}\left(\Gamma \mu+c_{\alpha q} \rho u_{\alpha}+\frac{3}{2 c^{2}}\left(c_{\alpha q} c_{\beta q}-\frac{3}{c^{2}} \delta_{\alpha \beta}\right) \phi u_{\alpha} u_{\beta}\right)
$$

while the distributions for $q=0$ are given by the following:

$$
\begin{aligned}
& f_{0}^{e q}=\rho-\sum_{q=1}^{Q-1} f_{q}^{e q} \\
& g_{0}^{e q}=\phi-\sum_{q=1}^{Q-1} g_{q}^{e q}
\end{aligned}
$$

The bulk pressure in Equation (14) obeys: $p_{0}=c_{S}^{2} \rho+\frac{A}{2} \phi^{2}-\frac{3 A}{4} \phi^{4}$.

The kinematic viscosity $v$ is related to $\phi$, which can be seen as follows:

$$
v(\phi)=v_{c} \frac{1-\phi}{2}+v_{d} \frac{1+\phi}{2}
$$


here $v_{c}$ and $v_{d}$ are kinematic viscosity of the continuous and dispersed phases, respectively. In this paper $v_{c}=v_{d}$.

For a planar interface at $x=0, \phi$ evolves over the interface as follows:

$$
\phi(x)=\tanh _{\bar{\xi}}^{x}
$$

where $\phi= \pm 1$ is the value of $\phi$ in bulk phase at either side of the interface.

The width of diffuse interface $\xi$ is given by the following ${ }^{[37]}$ :

$$
\xi=\left(\frac{2 \kappa}{-A}\right)^{1 / 2}
$$

And the surface tension $\sigma$ follows from ${ }^{[37]}$ :

$$
\sigma=\frac{4}{3}\left(\frac{-A}{2 k}\right)^{1 / 2}
$$

We have to calculate the spatial gradients of $\phi$ to determine the equilibrium distributions. The stencils for gradients and Laplacian calculations are ${ }^{[41]}$ :

$$
\begin{aligned}
& \partial_{x}=\frac{1}{12 \Delta x}\left[\left(\begin{array}{ccc}
0 & 0 & 0 \\
-1 & 0 & 1 \\
0 & 0 & 0
\end{array}\right),\left(\begin{array}{ccc}
-1 & 0 & 1 \\
-2 & 0 & 2 \\
-1 & 0 & 1
\end{array}\right),\left(\begin{array}{ccc}
0 & 0 & 0 \\
-1 & 0 & 1 \\
0 & 0 & 0
\end{array}\right)\right] \\
& \nabla^{2}=\frac{1}{6 \Delta x^{2}}\left[\left(\begin{array}{lll}
0 & 1 & 0 \\
1 & 2 & 1 \\
0 & 1 & 0
\end{array}\right),\left(\begin{array}{ccc}
1 & 2 & 1 \\
2 & -24 & 2 \\
1 & 2 & 1
\end{array}\right),\left(\begin{array}{lll}
0 & 1 & 0 \\
1 & 2 & 1 \\
0 & 1 & 0
\end{array}\right)\right]
\end{aligned}
$$

where the left, middle, and right matrices are the slices of the stencil when $z=\Delta x, 0$, and $-\Delta x$, respectively.

Solve the discretized equations (5) by two steps:

$$
\begin{array}{ll}
\text { Collision step: } & f_{q}^{\prime}\left(r_{\alpha}, t\right)=f_{q}\left(r_{\alpha}, t\right)-\frac{1}{\tau_{f}}\left(f_{q}-f_{q}^{e q}\right)+F_{q} \\
& g_{q}^{\prime}\left(r_{\alpha}, t\right)=g_{q}\left(r_{\alpha}, t\right)-\frac{1}{\tau_{g}}\left(g_{q}-g_{q}^{e q}\right) \\
\text { Streaming step: } \quad & f_{q}\left(r_{\alpha}+c_{\alpha q} \Delta t, t+\Delta t\right)=f_{q}^{\prime}\left(r_{\alpha}, t\right) \\
& g_{q}\left(r_{\alpha}+c_{\alpha q} \Delta t, t+\Delta t\right)=g_{q}^{\prime}\left(r_{\alpha}, t\right)
\end{array}
$$

\subsection{Scaling procedure}

The LBM works in lattice space, which means the parameters should be translated into lattice units $[\mathrm{lu}]$. Scaling factors are utilized to realize it. In this work, the parameters with represent those in lattice units.

The scaling factor for surface tension can be written as: 


$$
C_{\sigma}=\frac{\sigma}{\tilde{\sigma}}
$$

There are two numerical parameters related to surface tension, $\kappa$ and $A$. The equation for $\kappa$ is derived from Equation (20):

$$
\kappa=\frac{3 \xi \sigma}{4 C_{\sigma}}
$$

In this work the interface thickness $\xi$ (see Equation (19)) was 1.14 [lu] for each simulation. ${ }^{[36]}$ The value of $A$ can be calculated using Equation (19) once $\kappa$ has been determined.

The density of liquid is $\tilde{\rho_{c}}=1$; and we can obtain the density of droplet according to density ratio: $\tilde{\rho_{d}}=\lambda \tilde{\rho_{c}}$.

Since the density difference is much smaller than actual densities of the liquids, the density differences only influence the forcing term $\Delta \rho \mathrm{g}$ in Equation (1b). This is known as the Boussinesq approximation. The scaling factors for the forcing is based on the Eötvös number. According to the equation of Eo, forcing term becomes:

$$
\widetilde{\Delta \rho g}=\frac{\tilde{\sigma}}{\tilde{d}^{2}} E o
$$

where $\tilde{d}$ is the equivalent drop diameter.

In a similar way, based on the equation of $M o$ and $\eta$, the dynamic viscosity is defined as:

$$
\tilde{\mu_{c}}=\left(\tilde{\rho}_{c}^{2} \tilde{\sigma}^{2} \tilde{d}^{2} \frac{M o}{E o}\right)^{1 / 4} \quad \tilde{\mu_{d}}=\eta \tilde{\mu}_{c}
$$

And the kinematic viscosities are:

$$
\tilde{v_{c}}=\frac{\tilde{\mu_{c}}}{\tilde{\rho_{c}}} \quad \tilde{v_{d}}=\frac{\tilde{\mu_{d}}}{\tilde{\rho_{d}}}
$$

From the above, the following scaling factors have been introduced and can be determined:

$$
\begin{aligned}
& \text { surface tension: } C_{\sigma}=\frac{\sigma}{\tilde{\sigma}} ; \\
& \text { density: } C_{\rho}=\frac{\rho}{\tilde{\rho}} ; \\
& \text { kinematic viscosity: } C_{\nu}=\frac{v_{c}}{\tilde{v}_{c}} ; \\
& \text { forcing: } C_{\text {force }}=\frac{\Delta \rho \mathrm{g}}{\widetilde{\Delta \rho \mathrm{g}}} .
\end{aligned}
$$

Utilizing the equations of $F r, R e$, and $W e$, as well as the scaling factors (30), other 
scaling factors can be calculated as:

$$
\begin{aligned}
& \text { length: } C_{d}=\frac{C_{v}^{2} C_{\rho}}{C_{\sigma}} ; \\
& \text { velocity: } C_{u}=\frac{C_{\sigma}}{C_{v} C_{\rho}} ; \\
& \text { time: } C_{t}=\frac{C_{v}^{3} C_{\rho}^{2}}{C_{\sigma}^{2}} .
\end{aligned}
$$

The other parameters can be translated from physical units into lattice units according to the scaling factors.

\section{VERIFICATION OF SIMULATIONS}

The computer code for these simulations has been developed using FORTRAN 90. It is based on our previous study. ${ }^{[42]}$

The objective of the present study is to investigate the behaviour of rising droplet being sliced by using the above described LBM model. Several factors that may affect the process should be checked in advance. The results of a set of test simulations facilitated determining the suitable input parameters $(E o, M o, \lambda, \eta)$ as well as the associated numerical settings. In this section, results of verification computations for domain size, mesh resolution and dimensionality (2D versus 3D) are presented.

\subsection{Domain size influence}

We used fully periodic boundary conditions for describing a single drop moving through a large volume of fluid. We therefore need to make sure that the simulation domain is large enough so that the drop does not interact with itself and with the flow it creates over the periodic boundaries. On the other side, the domain size should be set reasonably to avoid excessive simulation time.

Two-dimensional simulations of system with physical properties as given in Table 1 and drops of $d=1.0 \mathrm{~mm}$ were performed. The heights of the computational domain were set to $8 d$ to $16 d$ to determine the proper height. The width is equal to $W=3 d$, and $D=1[\mathrm{lu}]$ (2D simulations). All computations were performed with a drop diameter in lattice units of $\tilde{d}$ $=30[\mathrm{lu}]$. The simulation parameters (in lattice units) were determined according to the scaling procedure as outlined in Section 3.3 after it was established that the physical system 
has $E o=1.0, M o=1.24 \times 10^{-4}, \eta=1$, and $\lambda=0.90$.

The results are shown in Figure 2 in terms of the Reynolds numbers and the vertical locations towards reaching a steady speed.
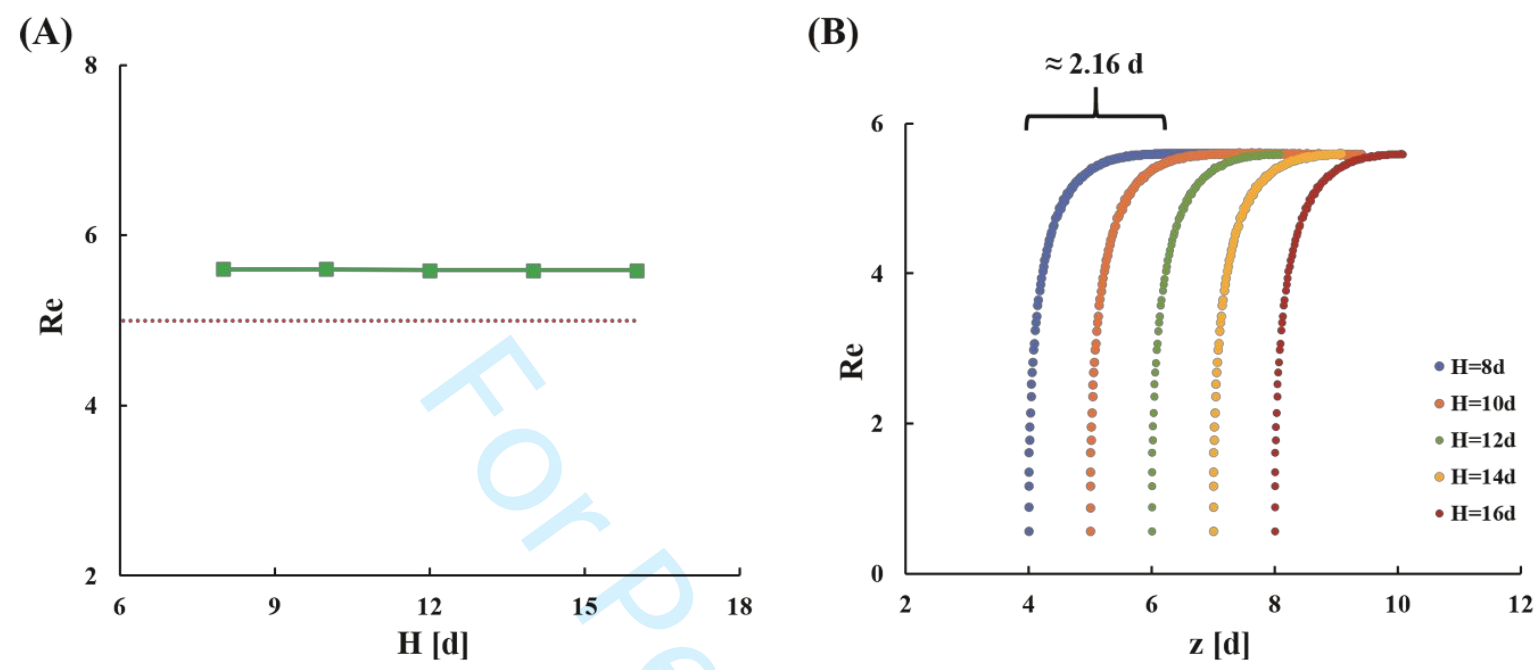

Figure 2. The results for a $d=1.0 \mathrm{~mm}$ drop for different simulation domain heights in terms of the steady-state Reynolds number and rising distance. The domain width is $3 d$, and depth is $1[\mathrm{lu}] ; E o=1.0$ and $M o=1.24 \times 10^{-4}$. The dashed line in the left panel indicates the Reynolds number as derived from Clift et al ${ }^{[12]}$

The steady state Reynolds numbers reported in Figure $2 \mathrm{~A}$ are within $0.2 \%$ of $R e=5.6$ so that the impact of height on rising velocity can be negligible, which is consistent with the three-dimensional simulation. ${ }^{[28]}$ The flow map due to Clift et al[12] indicates a Reynolds number of 5.0 for the specific values for $E o$ and $M o$ where it should be noted that this map is based on (three-dimensional) experimental data.

The choice for the height $H$ in the slicing simulations is mainly determined by the distance it takes for the drop to reach a steady speed. Figure $2 \mathrm{~B}$ shows that this distance is approximately $2.16 \mathrm{~d}$. Because the drop is released at $z>0$ and to ensure the drop behaviour has fully developed well before the drop hits the knife, a much larger domain height is used in the slicing simulations: $H=20 \mathrm{~d}$.

The verification simulations for the domain width were carried out in the range $W=2 d$ to $10 \mathrm{~d}$. The other domain parameters are $H=10 d, D=1$ [lu] (for two-dimensional 
simulations) or $W=D=2 d$ to $5 d$ (for three-dimensional simulations). All computations were performed with drop diameter $\tilde{d}=30[\mathrm{lu}]$.

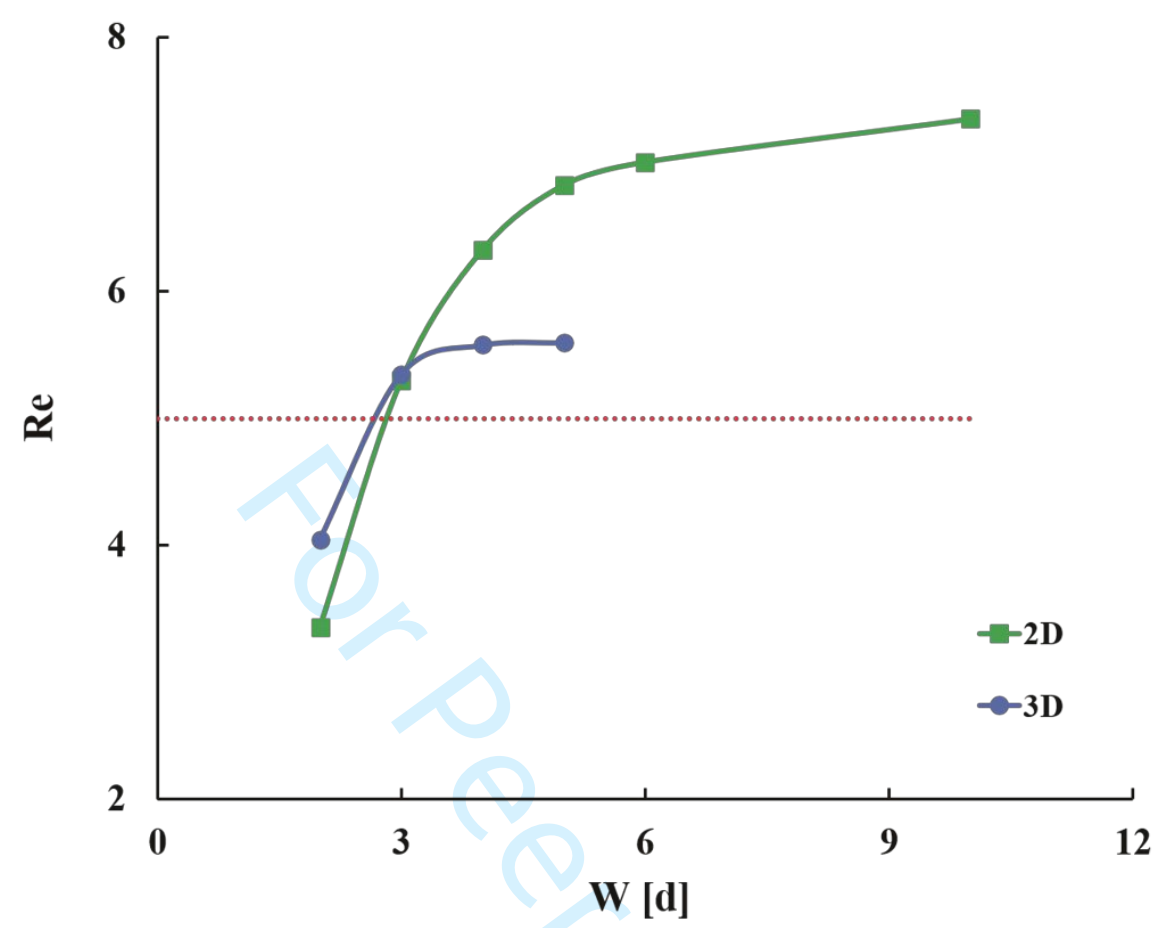

Figure 3. The results for a $d=1.0 \mathrm{~mm}$ drop for different simulation domain widths in terms of the steady-state Reynolds number. The domain height is $H=10 d$, and depth is 1 [lu] (for two-dimensional simulations) or $W$ (for three-dimensional simulations); $E o=1.0$ and $M o=1.24 \times 10^{-4}$. The horizontal dashed line indicates the Reynolds number as derived from Clift et al ${ }^{[12]}$

From comparing Figure 2 and 3 it is clear that the width of the domain has a much stronger influence on the rise velocity than the domain height. In three dimensions the rise velocity becomes independent of the domain width beyond $W=D=4 d$. Since $H=8 d$ in Figure 2, these results are also independent of domain height. The converged Reynolds number in the $3 \mathrm{D}$ results in Figure 3 is $R e=5.3$ and is close to the Reynolds number $R e=5.0$ that can be derived from the Clift et al[12] flow map.

Compared to the $3 \mathrm{D}$ results, the $2 \mathrm{D}$ results show a higher converged Reynolds number. This means the drag on a 2D (cylindrical) droplet is smaller than on a 3D (spherical) droplet. This has its analogy in flow around solid spheres and cylinders that for $R e<30$ shows 
slightly smaller drag coefficients for cylinders than for spheres.

\subsection{Mesh resolution}

The drop diameter $\tilde{d}$ is a measure for the spatial resolution of the simulation and therefore has impact on the computational effort. It may play an important role in the accuracy of the result. For this we here present results for a drop of $d=1 \mathrm{~mm}$ represented by $\tilde{d}=20$ to $60[\mathrm{lu}]$ and with $E o=1$ and $M o=1.24 \times 10^{-4}$.

Changing the diameter in lattice units implies rescaling simulation parameters. The idea is to change the drop diameter $\tilde{d}$ by a factor $f_{m}\left(\tilde{d}_{2}=f_{m} \tilde{d}_{1}\right)$. It is important to keep the dimensionless numbers constant, as well as viscosity and interface thickness: $\tilde{\mu_{c 2}}=\tilde{\mu_{c 1}}, \tilde{\mu_{d 2}}$ $\tilde{\mu_{d 1}}, \quad \xi_{2}=\xi_{1}$. The following relations make sure the Eötvös and Morton numbers are unchanged upon rescaling:

$$
\frac{\tilde{\sigma}_{2}}{\tilde{\sigma}_{1}}=\frac{1}{f_{m}} \quad \frac{(\widetilde{\rho \rho} g)_{2}}{(\Delta \widetilde{\rho} g)_{1}}=\frac{1}{f_{m}^{3}} \quad \frac{\kappa_{2}}{\kappa_{1}}=\frac{1}{f_{m}} \quad \frac{A_{2}}{A_{1}}=\frac{1}{f_{m}}
$$

The analysis was performed with simulations of $1.0 \mathrm{~mm}$ droplets with domain size $10 \tilde{d}$ $\times 3 \tilde{d} \times 3 \tilde{d}$ in $3 \mathrm{D}$ simulations and $10 \tilde{d} \times 3 \tilde{d} \times 1$ in $2 \mathrm{D}$ simulations. The resulting Reynolds numbers related to steady-state rise velocities are plotted in Figure 4. We observe good grid convergence behaviour for $2 \mathrm{D}$ as well as for $3 \mathrm{D}$ simulations. In the subsequent section on droplet slicing, a mesh resolution of $\tilde{d}=30[\mathrm{lu}]$ for $1 \mathrm{~mm}$ drops is adopted. In order to avoid extensive computations, 2D domains have been used in the simulation of droplets sliced by a knife. In Figures 3 and 4, we can see a reasonable agreement in terms of the Reynolds number based on the rise velocity between 2D and 3D. 


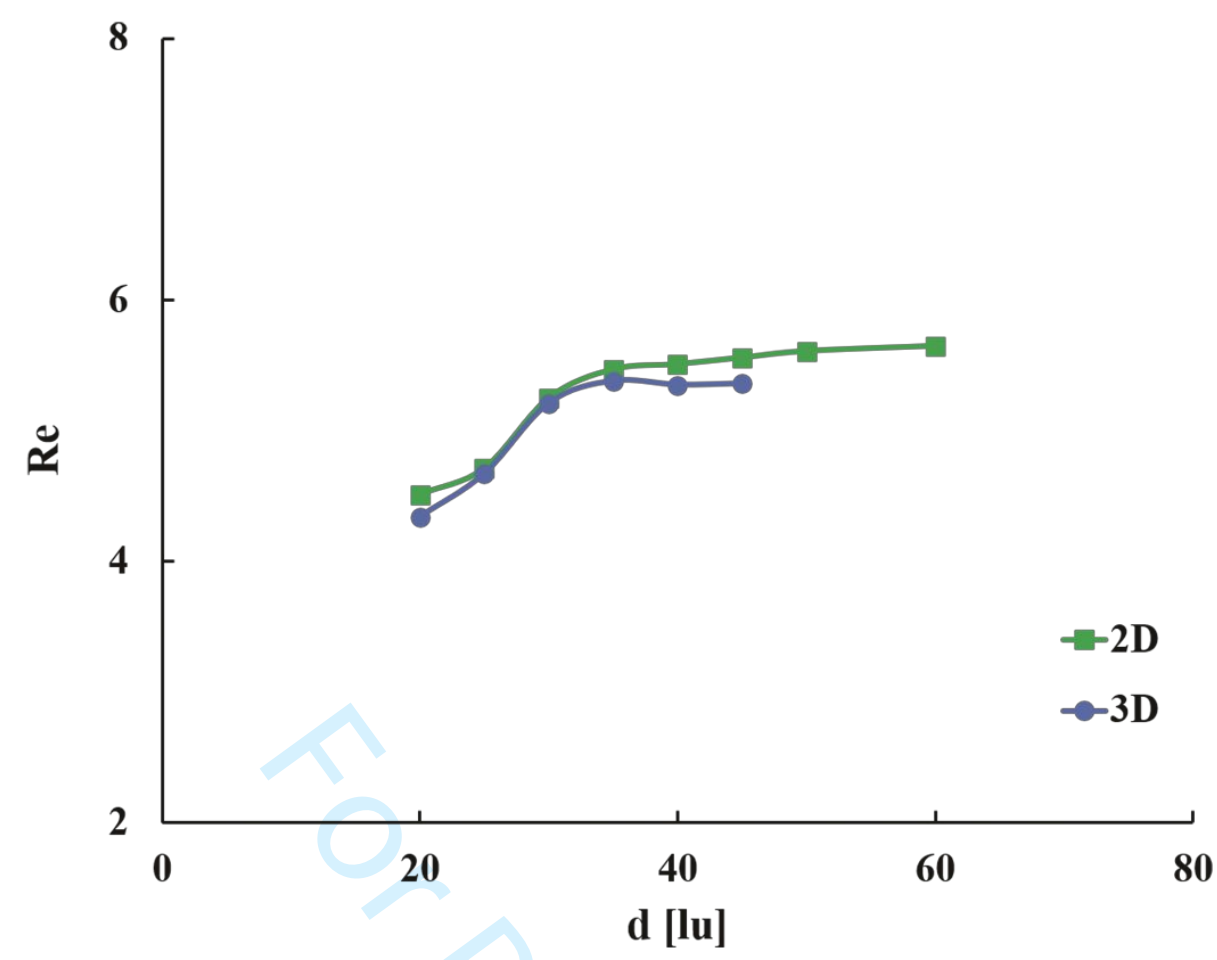

Figure 4. The results for a $d=1.0 \mathrm{~mm}$ drop for different drop diameter $\tilde{d}$ in lattice space in terms of steady-state Reynolds number; $E o=1.0$ and $M o=1.24 \times 10^{-4}$

With $\tilde{d}=30 \quad[\mathrm{lu}]$ (and $d=1 \mathrm{~mm}$ ), the scaling factor of surface tension is $C_{\sigma}=$ $1.0 \mathrm{~N} / \mathrm{m}$. It gives the linear dimension scale factor $C_{d}=3.33 \times 10^{-5} \mathrm{~m}$, time scale factor $C_{t}=4.66 \times 10^{-6} \mathrm{~s}$.

\section{RESULTS}

In this work, the parameters for simulated system are: $\lambda=0.9, \eta=1, M o=1.24 \times$ $10^{-4}$, the values of Eo are different for different drop diameters, see Table 2. According to the diagram of Clift et al, ${ }^{[12]}$ the drops are in the spherical (lower Eo) to ellipsoidal regime. Note that this diagram reflects a three-dimensional situation.

Table 2. Drop diameter $d$ and corresponding Eo number considered in the present simulations

\begin{tabular}{cc}
\hline$d(\mathrm{~mm})$ & Eo \\
\hline 1.0 & 1.00 \\
\hline
\end{tabular}




\begin{tabular}{rc}
\hline 2.0 & 3.96 \\
3.0 & 8.91 \\
4.0 & 15.84 \\
\hline
\end{tabular}

\subsection{The knife is at the centre}

The numerical simulations of slicing rising droplets have been carried out for $d=1.0-4.0 \mathrm{~mm}$ droplets, in order to observe the different slicing scenarios. For each droplet, the "approach velocity", deformation, breakage and slicing results have been analyzed. The "approach velocity" is the velocity at the moment the drop starts feeling the knife.

A range of scenarios has been observed when the drop hits the knife at centre and it has been investigated how the type of scenario depends on the dimensionless parameters governing the motion and slicing of the drop: the Eötvös number and the ratio of the droplet size and the width of knife.

The rising velocities for drops with diameter $1.0 \mathrm{~mm}$ to $4.0 \mathrm{~mm}$ as a function of the vertical $(z)$ location are depicted in Figure 5. The rising velocities of drops increase rapidly during the accelerating regime. Then, the 1.0 and $2.0 \mathrm{~mm}$ drops enter steady regimes. The velocity of $1.0 \mathrm{~mm}$ drop decreases rapidly when it hits the knife and the drop finally stops moving. The knife hinders the $2.0 \mathrm{~mm}$ drop in rising, and finally slices the drop into two parts. A different behaviour is noticed for $3.0 \mathrm{~mm}$ and $4.0 \mathrm{~mm}$ drops: oscillations appear both for drop shapes (see Figure 6) and rising velocities (Figure 5) over the rising period. The acceleration period becomes longer with the increase of drop diameter. At the same time, the amplitude of velocity and shape oscillation becomes larger. 


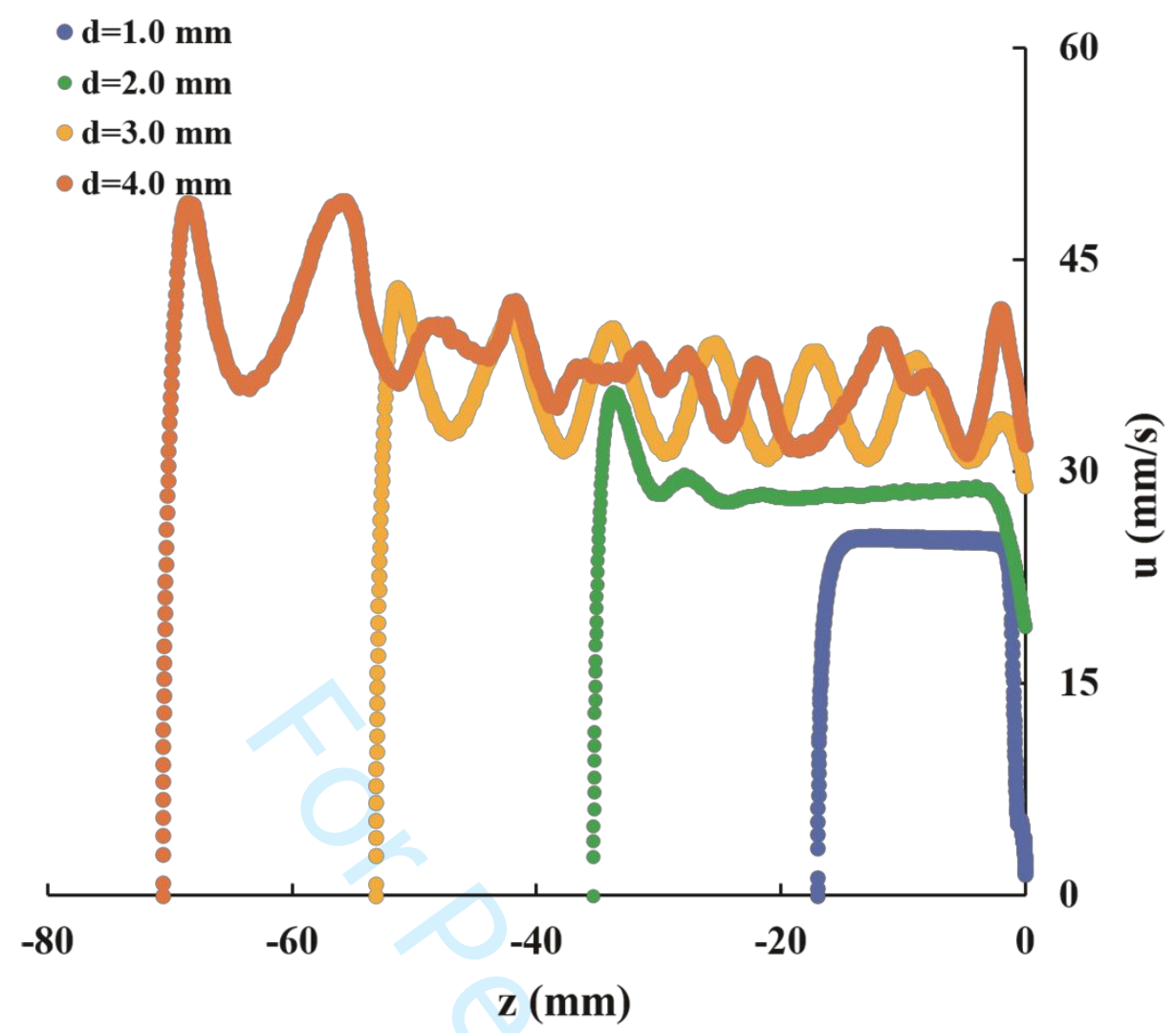

Figure 5. The rising velocity of drops

An increase in drop diameter results in obvious deformation in early times and leads to the oscillation behaviour of drop rising velocity. The drop shapes are presented in Figure 6 for 2.0, 3.0 and $4.0 \mathrm{~mm}$ drops. The drop deformations at initial moment are different, at $t=0.1$ $\mathrm{s}$, the shapes of droplets are similar in the three cases. Whereas, at $t=0.3 \mathrm{~s}$, the drops deform in different ways. The $2.0 \mathrm{~mm}$ drop changes its shape to ellipse after deformation, and maintains this shape until it touches the knife. At the same time, the larger drops (with diameter equals to $3.0 \mathrm{~mm}$ and $4.0 \mathrm{~mm}$ respectively) get convex shapes after which shape oscillations appear. The $4.0 \mathrm{~mm}$ drop develops two "tails" that then they break away and form two small satellite droplets. Surprisingly, during the ascent, they merge with the mother droplet again. 


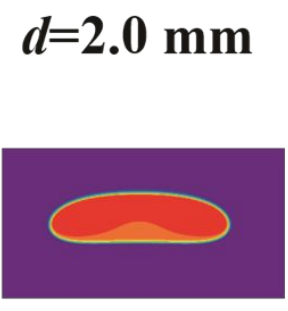

$$
t=1.0 \mathrm{~s}
$$

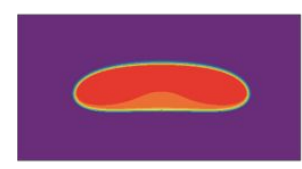

$t=0.8 \mathrm{~s}$

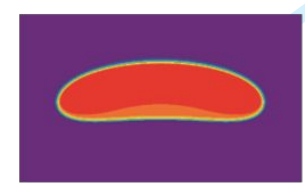

$t=0.3 \mathrm{~s}$

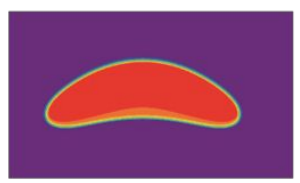

$t=0.15 \mathrm{~s}$

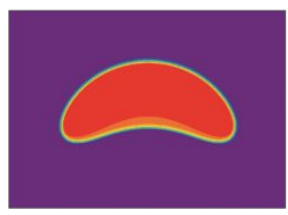

$t=0.1 \mathrm{~s}$

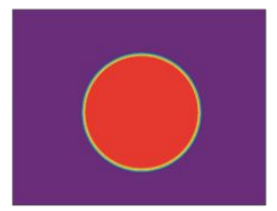

$t=0.02 \mathrm{~s}$ $d=3.0 \mathrm{~mm}$

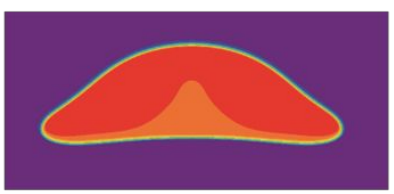

$t=1.2 \mathrm{~s}$

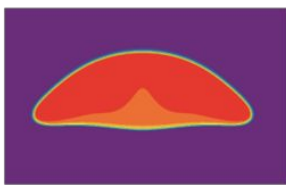

$t=0.8 \mathrm{~s}$

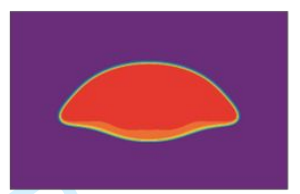

$t=0.3 \mathrm{~s}$

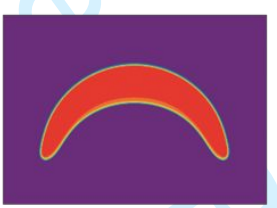

$t=0.15 \mathrm{~s}$

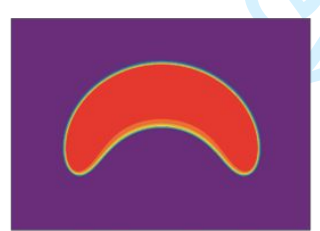

$t=0.1 \mathrm{~s}$

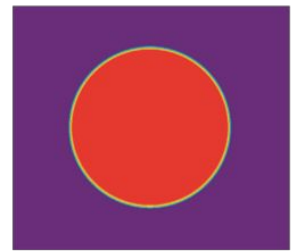

$t=0.02 \mathrm{~s}$ $d=4.0 \mathrm{~mm}$

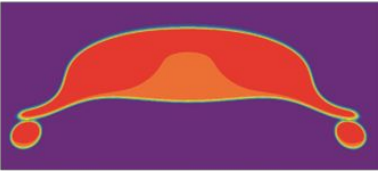

$t=1.8 \mathrm{~s}$

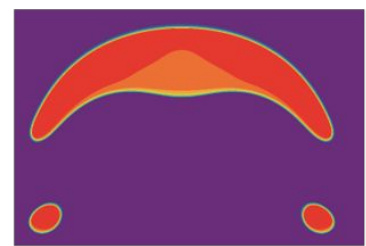

$t=0.8 \mathrm{~s}$

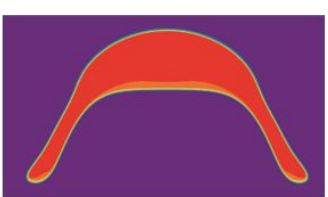

$t=0.3 \mathrm{~s}$

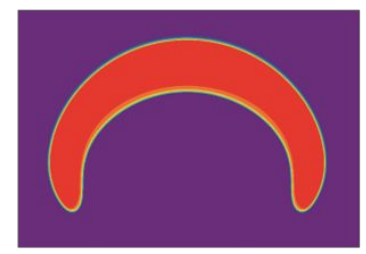

$t=0.15 \mathrm{~s}$

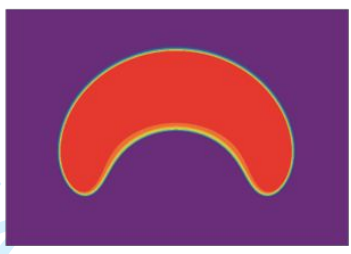

$t=0.1 \mathrm{~s}$

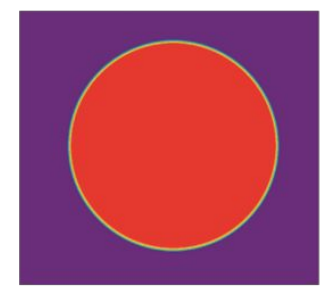

$t=0.02 \mathrm{~s}$

Figure 6. The shapes of two-dimensional droplets of $d=2.0,3.0$ and $4.0 \mathrm{~mm}$ at different moments after being released at $t=0$ and before hitting the knife

The "approach velocity" is related to drop diameter (see Figure 7). The aspect ratio $E$, 
which can be regarded as the ratio of the two principal axes of the droplet, is a description of the shape deformation quantitatively. $E=1$ means that the droplet has a circular shape. ${ }^{[43]}$ According to Michaelides, ${ }^{[43]}$ the droplet is a spherical if $E \geq 0.95$ (in this study, it is circular). Among the drop diameters involved in this study, only $d \leq 1.5 \mathrm{~mm}$ drop can be considered having a circular shape when it hits the knife. Droplets with larger diameters are either deformed or oscillating. The drops with diameter $d \geq 1.8 \mathrm{~mm}$ begin to deform, and $d \geq 2.8 \mathrm{~mm}$ begin to oscillate in shape and velocity. Besides, it is also found that the "approach velocity" for 1.8-2.6 mm get reduced, which may be caused by deformation; the ellipse-shaped drops are subject to greater resistance due to their greater width.

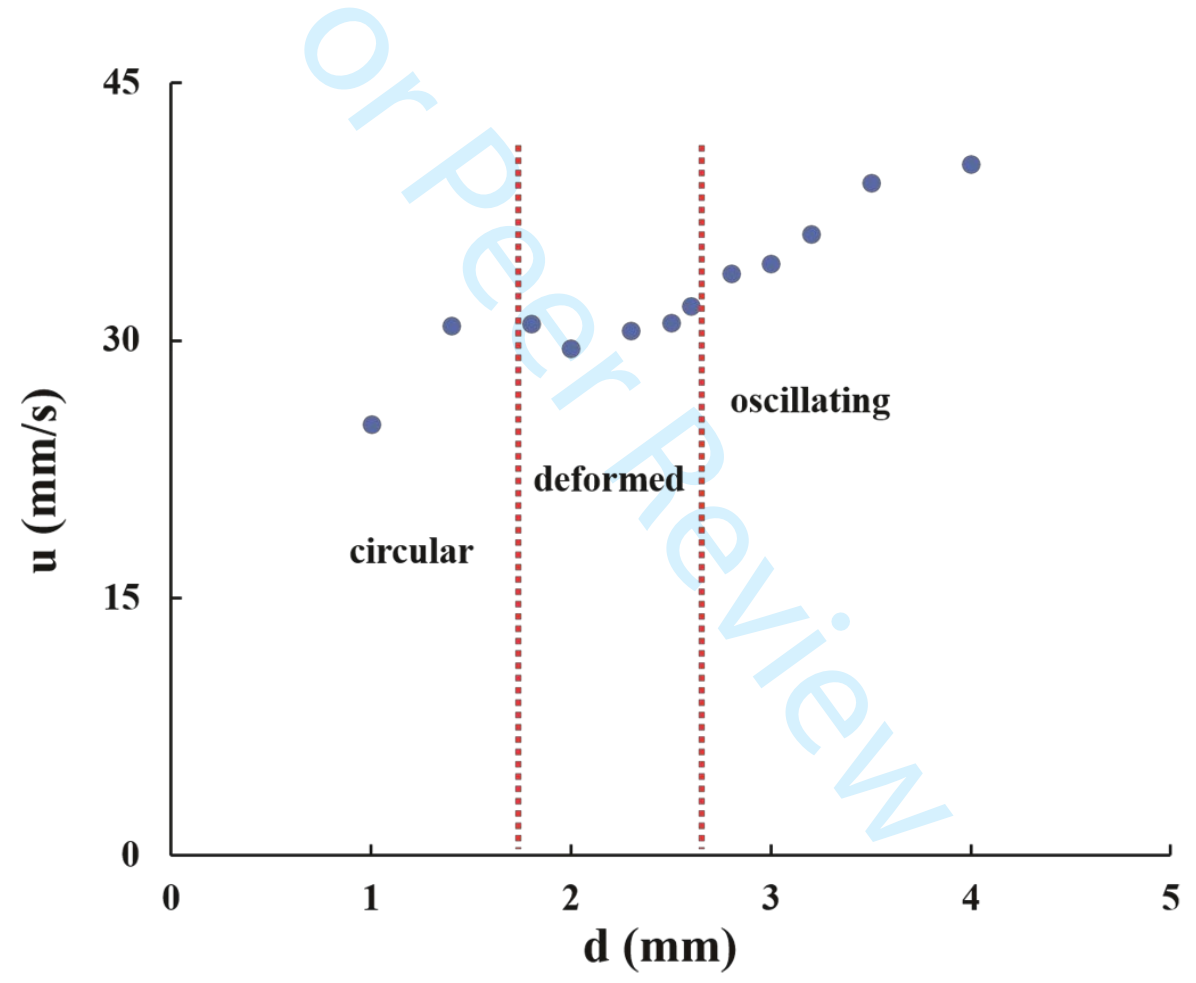

Figure 7. "Approach velocities" as a function of drop diameters

The slicing behaviour is different for different drop sizes. The smallest droplet of $d=1.0$ $\mathrm{mm}$ stops on the knife when sliced by knife, whether it is a sharp or a more obtuse knife (Figure 8A, B). Larger drops are easier to be sliced, and the slicing results do not depend much on the knife width (Figure $8 \mathrm{C}-\mathrm{G}$ ). 


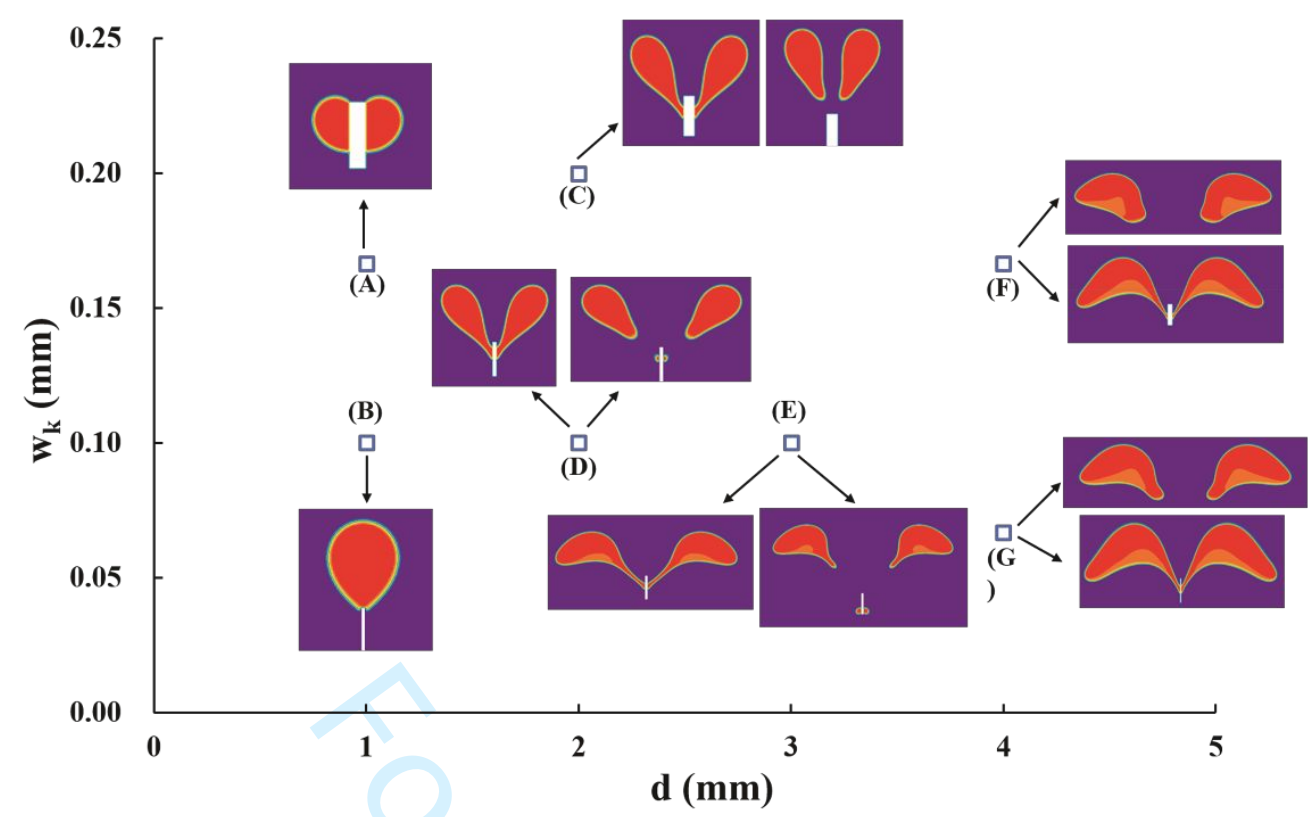

Figure 8. Slicing scenarios (identified by letters $(A)-(G)$ that are used in the text) as a function of drop size $d$ and width of the knife $w_{k}$

The effects of the relative sharpness of the knife $s_{r}$ have been studied. It is defined as:

$$
S_{r}=\frac{w_{k}}{d}
$$

where $w_{k}$ is the width of knife, $d$ is the drop diameter. The simulated data are plotted in the graphical correlation in Figure 9.

Figure 9 shows the relationship between $E o$ and the relative thickness of knife for $M o=1.24 \times 10^{-4}$. The results can be divided into three categories: (1) The droplet stops on the knife when sliced by relative blunt knife, this happens especially for smaller droplets (the lower Eo number). (2) The droplet passes the knife when it hits a sharp knife, and is not broken into two pieces. (3) The droplet is sliced into two parts for larger drop diameters / higher Eo.

The main conclusion of Figure 9 is that for a slicing process to be successful we need to overcome a threshold value of the Eotvos number of approximately $E o=4$. This critical value does not depend on the relative knife thickness, at least for $s_{r}<0.1$. 


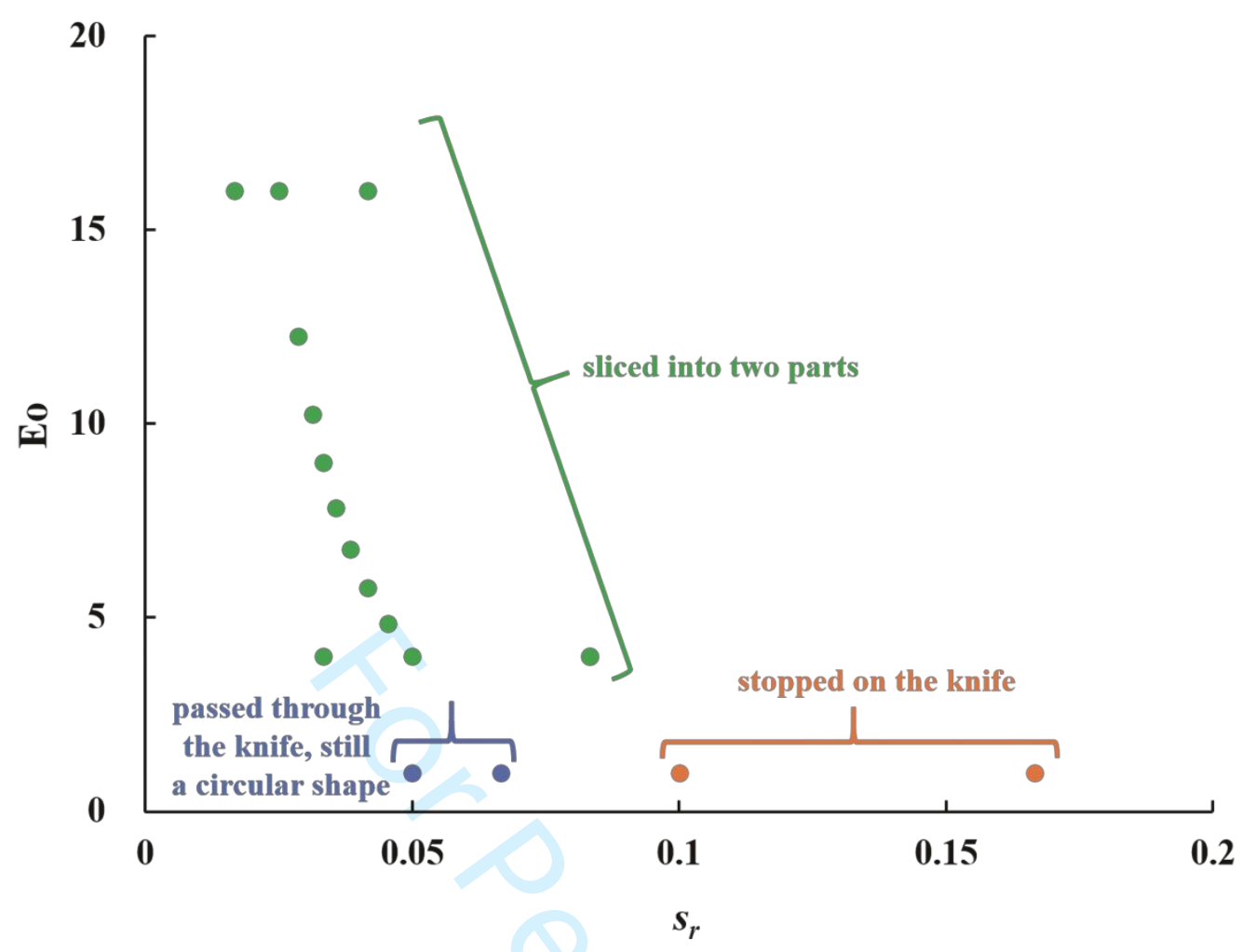

Figure 9. Map of slicing scenarios with on the horizontal axis the relative thickness of the knife $\left(s_{r}=\frac{w_{k}}{d}\right)$ and on the vertical axis the Eötvös number $(E o)$

\subsection{The knife is off-centre}

In this section, the scenarios have been studied when the drops hit the knife off centre. The size of the simulation domain is $20 \tilde{d} \times 3 \tilde{d} \times 1$, and the relative thickness of the knife is in the range $s_{r}=0.025-0.1$. The droplets are sliced into two unequal parts when the drop hits knife off centre. The situations before and after slicing are presented in Figure 10.

In a range of off-centre distances, the volume ratios (given the 2D nature of the slicing simulations, the term "volume ratio" is actually a surface ratio, i.e. a ratio of volumes per unit length in the $3^{\text {rd }}$ direction) for sliced drops increase with the deviation distances. Beyond this range, the drops also are sliced in two parts, but the smaller droplet remains on the knife and cannot escape (Figure 10, $r / d \geq 5 / 12$ for $d=2.0 \mathrm{~mm}$ ). We note in Figure 10 that under certain conditions the drops with $d=4.0 \mathrm{~mm}$ have developed satellites at the moment they interact with the knife. 


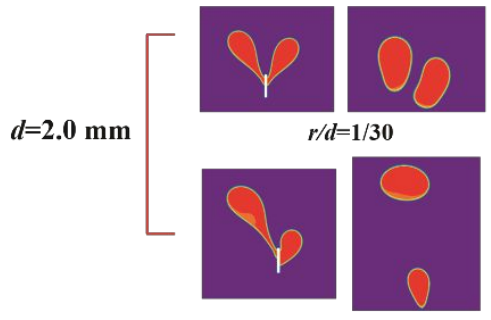

$r / d=0.2$

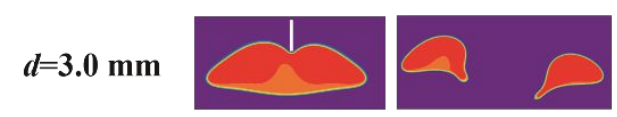

$r / d=1 / 18$

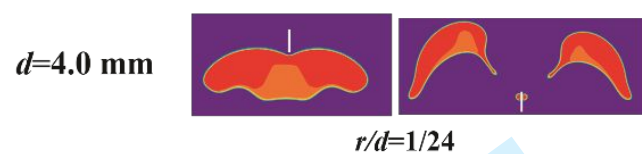

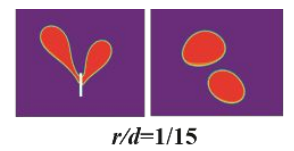

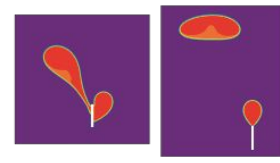

$r / d=5 / 12$

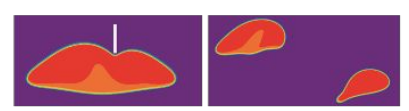

$r / d=1 / 6$

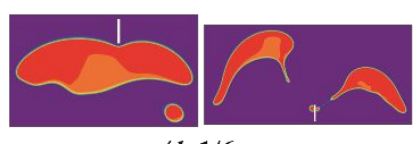

$r / d=1 / 6$
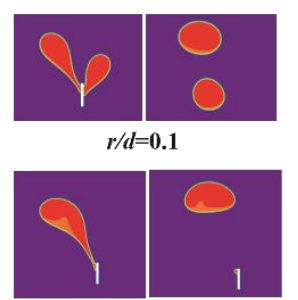

$r / d=5 / 6$
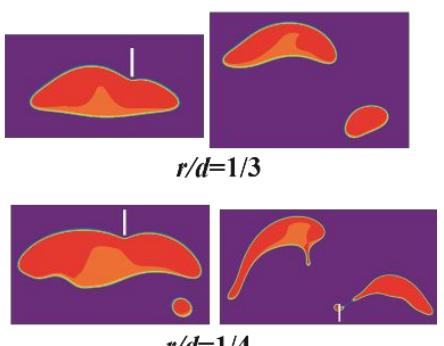

$r / d=1 / 4$

Figure 10. Slicing in case the drop approaches the knife off centre by a relative distance $r / d$ as indicated

The volume ratio of the two drops formed after slicing (the larger volume divided by the smaller volume) is a function of the relative off-centre distance $r / d$, see Figure 11 . The data points correlate well according to a quadratic relationship as can also be seen in Figure 11. The coefficients of the fitting equations are shown in Table 3, along with the $\mathrm{R}^{2}$ values that are all above 0.99 .

As expected, the volume ratio monotonically increases with the off-centre distance. However, the trend with respect to the drop diameter is not monotonic. The largest volume ratios are found for $d=3 \mathrm{~mm}$. Both $d=2 \mathrm{~mm}$ and $d=4 \mathrm{~mm}$ have smaller volume ratios for the same $r / d$ values. This effect is related to the shape of the drops at the moment they start interacting with the knife.

Table 3. The fitting equations between volume ratio (y) versus relative off-centre distance $r / d(\mathrm{x})$

\begin{tabular}{ccc}
\hline$d(\mathrm{~mm})$ & \multicolumn{2}{c}{ Fitting equation } \\
\hline 2.0 & $y=8.32 x^{2}+2.07 x+1.01$ & $\left(R^{2}=0.9995\right)$ \\
3.0 & $y=11.59 x^{2}+3.02 x+1.01$ & $\left(R^{2}=0.9999\right)$ \\
4.0 & $y=1.11 x^{2}+2.98 x+1.01$ & $\left(R^{2}=0.9934\right)$ \\
\hline
\end{tabular}




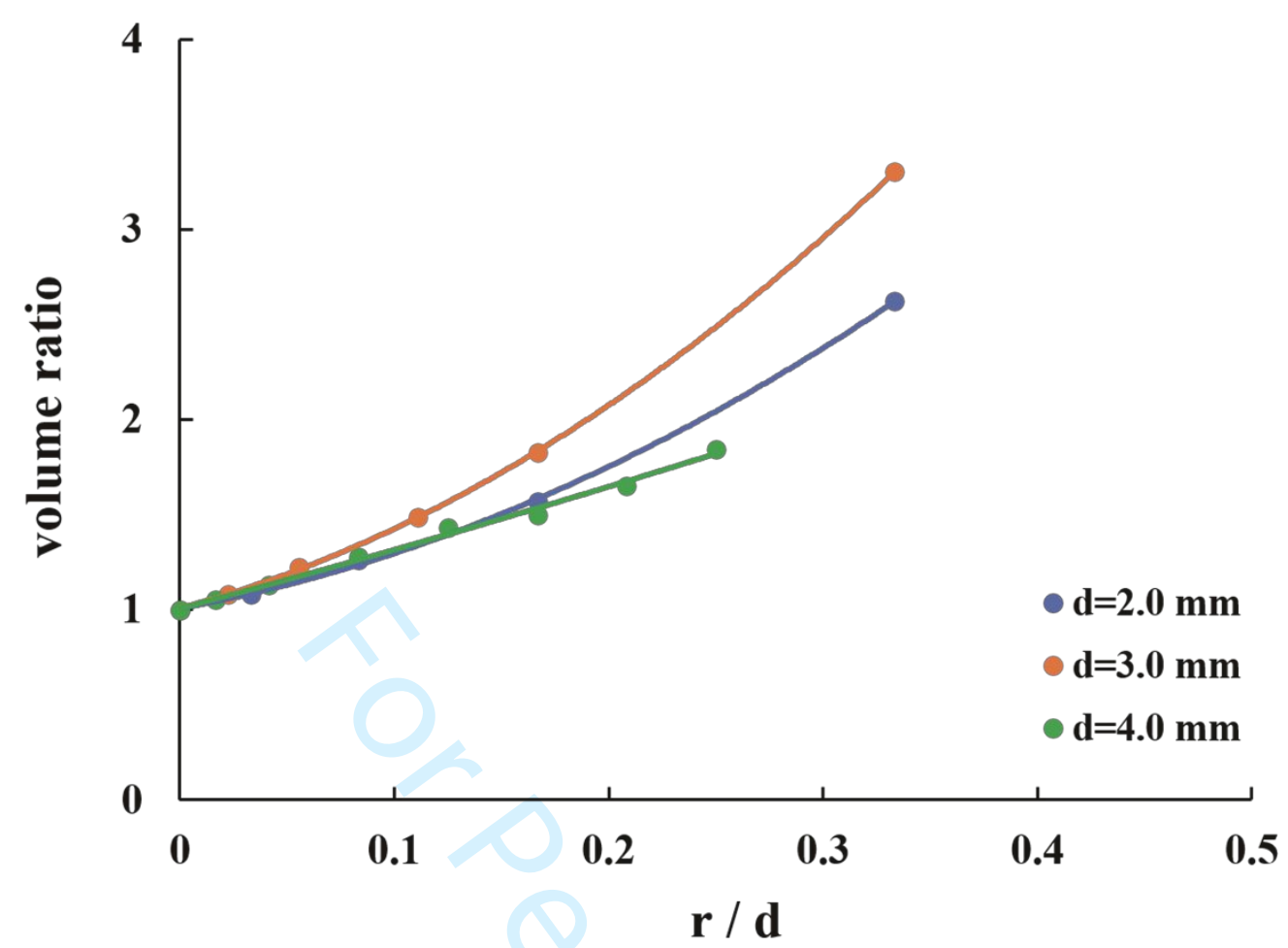

Figure 11. Ratio of the volume of the two drops formed after slicing as a function of the relative off-centre distance $r / d$. The dots represent individual simulations, the curves are quadratic fits (see Table 3)

\section{CONCLUSION}

In this work, we performed numerical simulations of slicing rising droplet in liquid. Two-dimensional and three-dimensional simulations have been performed using free energy LBM method. The proposed scaling procedure is utilized to translate the parameters into lattice units, which is corresponding to the physical units of the simulated system.

The simulation parameters such as simulation domain size, mesh resolution, and dimensionality (2D versus 3D) were tested to verify the LBM model and computer code. In order to make sure that the drop does not interact with itself and with the flow it creates over the periodic boundaries, the domain width should be at least three drop diameters. The mesh resolution also plays an important role in the accuracy of results. A diameter $\tilde{d}=30[\mathrm{lu}]$ for $1 \mathrm{~mm}$ is the best choice because it satisfies both accuracy and simulation time. The results of 
2D and 3D simulations were also compared. Two-dimensional slicing simulations have been adopted in order to avoid extensive computations.

We studied droplets with diameters of 1.0-4.0 mm, which were distributed in regions of circular, deformed, and oscillating. Our simulations captured shape deformation, rising velocity, and slicing results. The results of our numerical simulations (the rising velocity and Reynolds number) are consistent with the experimental data obtained by Clift et al. ${ }^{[12]}$

A range of scenarios have been observed when the drop hits the knife at the centre as well as off-centre. Droplet slicing requires a threshold Eotvos number. Our simulations suggest it to be $E o \approx 4$ if $M o=1.24 \times 10^{-4}$. This critical Eo was independent of the thickness of the knife as long as it was less than $0.1 d$. In case the drop hits the knife off-centre the volume ratio of the two resulting fragments depends approximately quadratically on the off-centre distance.

It should be noted that the above conclusions are for a viscosity ratio of one and a density ratio close to one. We anticipate that the main effect of the density ratio is an effect on the rise velocity which, through the Eotvos number, impacts slicing. As for the viscosity ratio: its impact is likely to be more complex, in line with a former paper ${ }^{[44]}$ on the effect of the viscosity ratio of drop breakage as a result of shear.

\section{ACKNOWLEDGEMENT}

This research was sponsored by Shanghai Sailing Program (No. 20YF1416000) and SUES Distinguished Overseas Professor Program. 


\section{NOMENCLATURE}

\begin{tabular}{|c|c|}
\hline$\rho$ & density $\left(\mathrm{kg} / \mathrm{m}^{3}\right)$ \\
\hline$\mu$ & dynamic viscosity (Pa s) \\
\hline$d$ & equivalent diameter $(\mathrm{mm})$ \\
\hline$\Delta \rho$ & density difference $\left(\mathrm{kg} / \mathrm{m}^{3}\right)$ \\
\hline$t$ & time $(s)$ \\
\hline$\sigma$ & interfacial tension $(\mathrm{N} / \mathrm{m})$ \\
\hline$u$ & rising velocity $(\mathrm{mm} / \mathrm{s})$ \\
\hline Eo & (dimensionless) Eötvös number \\
\hline Mo & (dimensionless) Morton number \\
\hline$\lambda$ & density ratio \\
\hline$\eta$ & dynamic viscosity ratio \\
\hline$R e$ & (dimensionless) Reynolds number \\
\hline$W e$ & (dimensionless) Weber number \\
\hline $\mathrm{Fr}$ & (dimensionless) Froude number \\
\hline$\phi$ & order parameter of phase field \\
\hline $\boldsymbol{r}$ & position vector $(\mathrm{mm} / \mathrm{s})$ \\
\hline$v$ & kinematic viscosity $\left(\mathrm{m}^{2} / \mathrm{s}\right)$ \\
\hline$g_{\alpha}$ & gravitational acceleration $\left(\mathrm{m} / \mathrm{s}^{2}\right)$ \\
\hline$M$ & mobility parameter \\
\hline$\Gamma$ & coefficient of mobility \\
\hline$P_{\alpha \beta}^{\text {th }}$ & "thermodynamic" pressure tensor $(\mathrm{Pa})$ \\
\hline$P_{\alpha \beta}^{\text {chem }}$ & “chemical” pressure tensor $(\mathrm{Pa})$ \\
\hline$P_{\alpha \beta}$ & full pressure tensor $(\mathrm{Pa})$ \\
\hline$\mu(\phi)$ & chemical potential $(\mathrm{Pa})$ \\
\hline$f(\boldsymbol{r}, t), g(\boldsymbol{r}, t)$ & particle distribution functions \\
\hline$q$ & discretized velocity direction \\
\hline$f_{q}^{e q}, g_{\mathrm{q}}^{\mathrm{eq}}$ & discrete Maxwell-Boltzmann distributions (or equilibrium distributions) \\
\hline$c_{\alpha q}$ & discrete velocity $(\mathrm{mm} / \mathrm{s})$ \\
\hline$\tau_{f}, \tau_{g}$ & dimensionless relaxation parameters \\
\hline
\end{tabular}




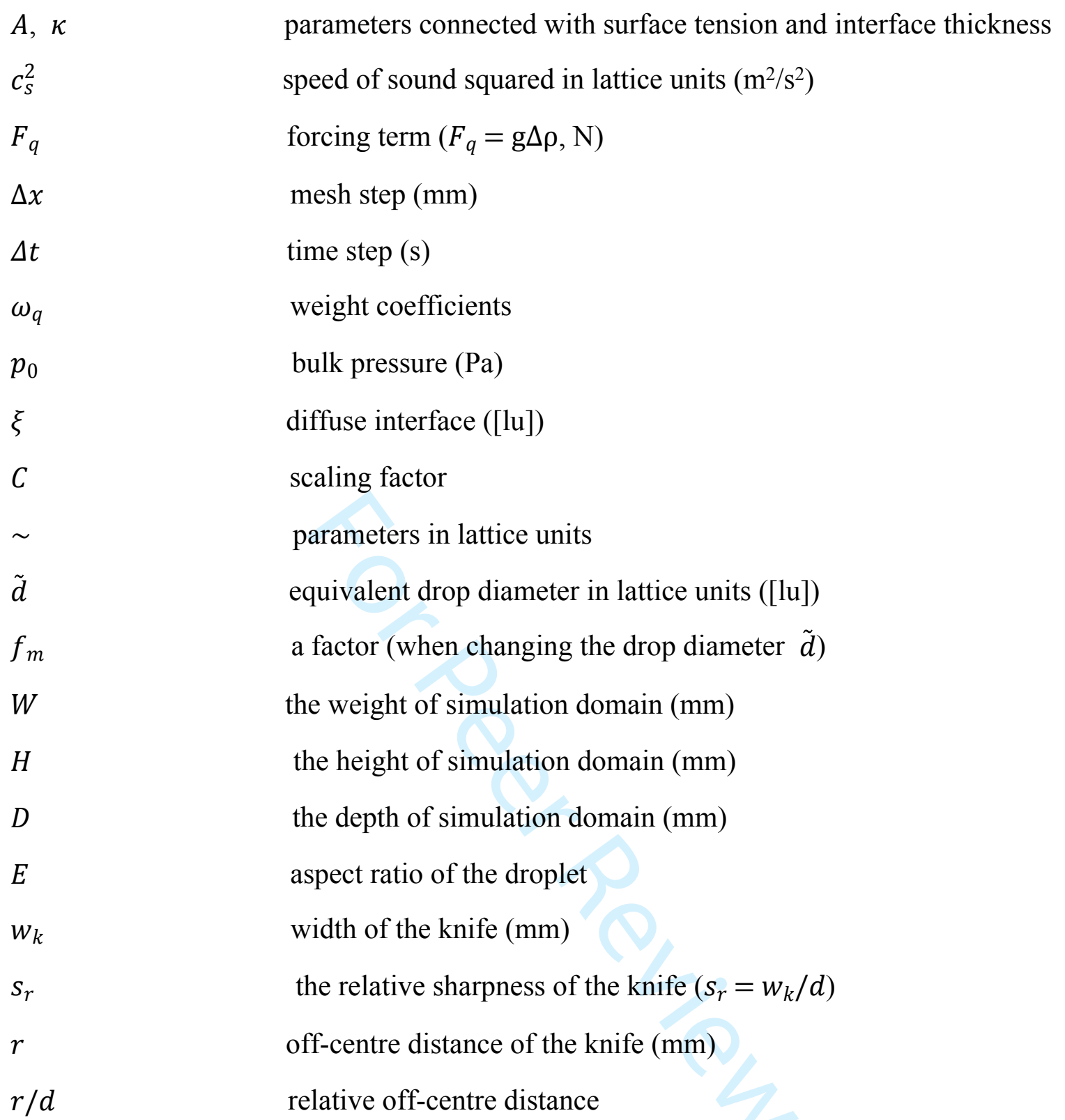




\section{REFERENCES}

1. K. K. Arun, K. Gibum, C. Wonjae, M. M. Joseph, T. Anish, Nat. Commun. 2012, 3, 1.

2. G. Zhang, M. Li, B. Zhang, J. Mater. Chem. A 2014, 2, 15284.

3. J. Li, X. Bai, X. Tang, F. Zha, H. Feng, W. Qi, Sep. Purif. Technol. 2017, 195, 232.

4. S. Lee, B. S. Ahn, J. Ind. Eng. Chem. 2003, 9, 768.

5. A. Carlescu, A. C. Blaga, A.-I. Galaction, M. Turnea, D. Cascaval, Sep. Sci. Technol. 2014, 49, 974.

6. C. Cheng, C. C. Alex, J. A. Yeh, Opt. Express 2006, 14, 4101.

7. M. Zhang, J. Li, W. Pei, Y. Liu, L. Zhong, S. Wang, Y. Zheng, Adv. Mater. Interfaces 2020, 7, 2000161.

8. E. O. Straume, R. E. M. Morales, A. K. Sum, Energy Fuels 2019, 33, 1.

9. G. Forte, E. Brunazzi, F. Alberini, Chem. Eng. Res. Des. 2019, 148, 417-428.

10. Z. Jaworski, P. Pianko-Oprych, D. L. Marchisio, A. W. Nienow, Chem. Eng. Res. Des. 2007, 85 (5), 753-759.

11. Y. Ren, K. S. Koh, M. Yew, J. K. Chin, Y. Chan, Y. Yan, Micromachines 2018, 9 (2), 57-74.

12. R. Clift, J. R. Grace, M. E. Weber, Bubbles, drops, and particles, 1st ed., Academic Press, New York 1978, p. 394.

13. B. Jie, S. Laura, Appl. Math. Model. 2013, 37, 1860.

14. D. Albernaz, M. Do-Quang, G. Amberg, Phys. Rev. E 2015, 91, 043012.

15. A. Begmohammadi, M. Farhadzadeh, M. H. Rahimian, Int. Commun. Heat Mass Transfer 2015, 61, 78 .

16. W. Wang, Z. Liu, Y. Jin, Y. Cheng, Chem. Eng. J. 2011, 173, 828.

17. L. Chen, Q. Kang, Y. Mu, Y.-L. He, W.-Q. Tao, Int. J. Heat Mass Transfer 2014, 76, 210.

18. L. Biferale, P. Perlekar, M. Sbragaglia, F. Toschi, Phys. Rev. Lett. 2012, 108, 104502.

19. Q. Li, K. H. Luo, Q. J. Kang, Y. L. He, Q. Chen, Q. Liu, Prog. Energy Combust. Sci. 2016, 52, 62 .

20. Z. Chai, T. Zhao, Acta Mech. Sin. 2012, 28, 983.

21. Y. B. Salah, Y. Tabe, T. Chikahisa, J. Power Sources 2012, 199, 85. 
22. S. Chen, G. D. Doolen, Annu. Rev. Fluid Mech. 1998, 30, 329.

23. A. Fakhari, M. H. Rahimian, AIP Conf. Proc. 2008, 1048, 727.

24. L.-B. Danie, K. H. Luo, R. Liu, P. Lv, Phys. Fluids 2014, 26, 023303.

25. H. M. Zhou, X. H. Yu, International Conference on Mechanics and Mechatronics, Changsha, March 2015.

26. E. Bertakis, S. Groß, J. Grande, O. Fortmeier, A. Reusken, A. Pfennig, Chem. Eng. Sci. 2010, 65, 2037.

27. T. Watanabe, K. Ebihara, Comput. Fluids 2003, 32, 823.

28. A. E. Komrakova, D. Eskin, J. J. Derksen, Phys. Fluids 2013, 25, 042102.

29. M. R. Swift, E. Orlandini, W. R. Osborn, J. M. Yeomans, Phys. Rev. E 1996, 54, 5041.

30. D. Jacqmin, J. Comput. Phys. 1999, 155, 96.

31. H. Ding, P. D. M. Spelt, C. Shu, J. Comput. Phys. 2007, 226, 1439.

32. P. Yue, J. J. Feng, C. Liu, J. Shen, J. Fluid Mech. 2004, 515, 293.

33. V. E. Badalassi, H. D. Ceniceros, S. Banerjee, J. Comput. Phys. 2003, 190, 371.

34. J. W. Cahn, J. E. Hilliard, J. Chem. Phys. 1958, 28, 258.

35. J. W. Cahn, J. E. Hilliard, J. Chem. Phys. 1959, 31, 688.

36. V. M. Kendon, M. Cates, I. Pagonabarraga, J.-C. Desplat, P. Bladon, J. Fluid Mech. 2001, 440, 147.

37. T. Kruger, H. Kusumaatmaja, A. Kuzmin, O. Shard, G. Silva, E. M. Viggen, The lattice Boltzmann method principles and practice, 1st ed., Springer, Switzerland 2017, p. 705.

38. A. Vorobev, Phys. Rev. E 2010, 82, 056312.

39. P. L. Bhatnagar, E. P. Gross, M. Krook, Phys. Rev. E 1954, 94, 511.

40. D. d'Humieres, I. Ginzburg, M. Krafczyk, P. Lallemand, L.-S. Luo, Philos. Trans. R. Soc. London, Ser. A 2002, 360, 437.

41. H. Kusumaatmaja, PhD thesis, University of Oxford, Oxford 2008.

42. A. E. Komrakova, O. Shardt, D. Eskin, J. J. Derksen, Int. J. Multiphase Flow 2014, 59, 24-43.

43. E. Michaelides, Particles, bubbles \& drops: Their motion, heat and mass transfer, 1 st ed., World Scientific, Hackensack 2006, p. 424.

44. A. E. Komrakova, O. Shardt, D. Eskin, J.J. Derksen, Chem. Eng. Sci. 2015, 126, 150-159. 